\title{
Structural Safety Evaluation of Tunnel Based on the Dynamic Monitoring Data during Construction
}

\author{
Xiaokun Yan $\left(\mathbb{D},{ }^{1}\right.$ Hu Li $\left(\mathbb{D},{ }^{2}\right.$ Feng Liu $\left(\mathbb{D},{ }^{2}\right.$ and Yang Liu $\mathbb{D}^{2}$ \\ ${ }^{1}$ Shenhua Institute of Transportation Technology Research, Beijing 100080, China \\ ${ }^{2}$ School of Transportation Science and Engineering, Harbin Institute of Technology, Harbin 150001, China \\ Correspondence should be addressed to Yang Liu; ly7628@hit.edu.cn
}

Received 23 November 2020; Revised 17 December 2020; Accepted 22 December 2020; Published 5 January 2021

Academic Editor: Mi-An Xue

Copyright (c) 2021 Xiaokun Yan et al. This is an open access article distributed under the Creative Commons Attribution License, which permits unrestricted use, distribution, and reproduction in any medium, provided the original work is properly cited.

It is still a challenge to accurately evaluate the structural safety of tunnel during the process of construction. To address this issue, a safety evaluation approach of tunnel based on the monitoring data during construction is proposed in this study. Firstly, the detailed description of modelling the tunnel excavation, releasing the load acting on the tunnel, and selecting the constitutive relationship of surrounding rock of tunnel is introduced. Secondly, aiming at an actual shallow-buried tunnel with underground excavation, utilizing the analytical results of deformation of tunnel, the structural safety of tunnel is evaluated by using a reliability-based method. Finally, the effectiveness of the proposed method is demonstrated by using the dynamic monitoring data obtained during the construction of an actual tunnel.

\section{Introduction}

With the rapid development of underground rail transit, the importance of tunnel engineering is gradually increasing. At present, there is a huge number of tunnel projects which are under construction, and the safety of tunnel construction is taking more and more attention, and the safety evaluation of tunnel construction structure becomes a burning problem. In the field of safety evaluation, there are many relevant research studies that have already been conducted by scholars. Prosek [1] combined deterministic safety analysis and probabilistic safety evaluation. They demonstrated the influence of deterministic safety analysis and integrated it into probabilistic safety evaluation. Chi et al. [2] studied the spatial risk factors of earth moving accidents, determined the spatial data requirements for automatic safety assessment based on existing safety regulations, and then they proposed an image-based safety assessment method for earth moving and surface mining activities. Lan et al. [3] selected seepage pressure, tidal level, and rainfall as safety evaluation indexes of seawall with the monitoring data. Using fuzzy theory, they established the safety evaluation method of seawall; after that, a method to determine safety grade of seawall based on comprehensive safety grade value was proposed. Yang [4] proposed a new concept of safety entropy, combined with functional resonance analysis method (FRAM), and applied it in the field of safety evaluation.

There are many scholars who made specific studies on tunnel construction. To find out the best construction method of long-span bifurcated tunnel, Xin and Gao [5] made a comparative analysis of six construction methods by using numerical simulation. Through field investigation, Zhang et al. [6] studied the landslide phenomenon caused by the excavation of a long-span multiarch tunnel from the aspects of tunnel section, construction defects, surrounding rock stability, and landslide conditions. Jin et al.[7] studied the construction technology considering the best excavation sequence in order to ensure the safety of large-span shallowburied tunnel construction.

The finite element method (FEM) is an important tool for tunnel research. Based on the theory of elastic-plastic, FEM calculates the stress field and deformation field of rock and soil under certain environmental conditions by solving simultaneous equations of elastic-plastic mechanics, and then the yield criterion of rock and soil is utilized to judge the state of surrounding rock [8-10]. This method can be 
used to solve the problems of elasticity and elastic-plastic in underground engineering; meanwhile, it can evaluate the stability of the whole tunnel structure and surrounding rock and guide the construction of tunnel, which could be widely used in practical engineering. And there are many scholars who have studied the application of FEM in tunnel engineering.

Yoshimura et al. [11] studied the surrounding rock deformation law during tunnel construction, comparing the actual monitoring data with the results calculated by FEM software, so as to verify the correctness of the FEM simulation. Sulem [12] took into full consideration about the rheological properties of surrounding rocks, the thrust distance of the palmtop, and the interaction between surrounding rocks and support, and then a prediction method for the displacement convergence of tunnel side walls was proposed. Abbo et al. [13] used the FEM analysis to study the undrained stability of rectangular tunnel. Yu et al. [14] used the FEM to simulate and analyze the seismic damage of key parts of the tunnel and studied the seismic response of the tunnel. Shahin et al. [15] studied the impact of tunnel excavation on the foundation of existing buildings. Alsahly et al. [16] developed a new computing framework and used the FEM to simulate the shield tunneling behavior. Ali et al. [17] used stochastic adaptive finite element limit analysis to study the influence of spatial variability on the undrained stability of circular tunnel without lining. Zheng et al. [18] proposed an Euler FEM to evaluate the stability of circular tunnels in undrained clay and conducted parametric analysis. Shiau et al. [19] used the numerical simulation to study the working face stability of circular tunnels under different soil mass and tunnel diameter-depth ratio.

The research of the above scholars has provided some support for the reasonable construction of the tunnel and a foundation for safety assessment, but there are still few research studies on the safety assessment methods of tunnel construction. The method using the continuous monitoring data is an effective method to assess the condition of structures in construction and operation [20-22]. Therefore, aiming at the problem of safety assessment of tunnel construction structure, a method of safety assessment of tunnel construction structure based on dynamic monitoring data is proposed. Firstly, the finite element modelling for the construction process of shallow-buried tunnel is analyzed. Then, the safety assessment method of tunnel construction structure based on reliability theory is proposed. Finally, the effectiveness of the proposed method is verified based on an actual tunnel.

\section{Structural Safety Evaluation of Tunnel Using the Reliability-Based Method}

2.1. Numerical Simulation of Tunnel Excavation with Finite Element Analysis. To simulate the process of tunnel excavation, the method based on FEM is described in three aspects, i.e., the tunnel excavation, releasing the load acting on the tunnel, and selecting the constitutive relationship of surrounding rock of tunnel, which provides the basis for further safety assessment for tunnel excavation.

\subsubsection{Simulation of the Process of Tunnel Excavation.} The key to simulate the excavation process of tunnel by numerical method is to simulate the gradual release of ground stress in tunnel construction. Before the tunnel excavation, the surrounding rock lies in a stable initial stress state due to long-term dead weight. The stresses in these boundaries are released due to the excavation of the tunnel and the application of support, which induce the redistribution of the stress field of the surrounding rock. The virtual support force release method usually is implemented to simulate the above process. The principle of this method is based on the actual construction situation, and the virtual support force inside the tunnel is utilized to release the in situ stress load step by step in order to simulate the tunnel construction process, as shown in Figure 1.

Before tunnel excavation, the initial stress of surrounding rock is $\sigma_{0}$, and the state of tunnel excavation without initial support is shown in Figure 1(b). Assume that, at this stage, part of the in situ stress load stored in the surrounding rock is released, i.e., $f_{1 i}$. The virtual support force $p_{1 i}$ is applied at the corresponding node position of the tunnel excavation boundary as the tunnel excavation. At this stage, the stress of surrounding rock is $\sigma_{1}$. Figure 1(c) shows the state of the circular tunnel after the initial support is applied. The red circle represents the initial support, and the released node load at this stage is $f_{2 i}$. The virtual support force applied is reduced to $p_{2 i}$, and the surrounding rock stress is defined as $\sigma_{2}$. Figure $1(\mathrm{~d})$ shows the state of the tunnel after the secondary lining is applied. The orange ring represents the secondary lining of the tunnel. At this time, there is no nodal force at the tunnel excavation boundary. The remaining node load can be completely released. In order to simulate this construction process, the virtual support force is reduced to zero, and then the obtained surrounding rock stress is $\sigma_{3}$.

The virtual support force gradually release method can simulate the continuous construction of tunnel construction process, and its calculation results do not need superposition of stress field and displacement field. The obtained displacement field and stress field are the actual stress field and displacement field after the completion of tunnel excavation. This method is in line with the actual situation of tunnel construction.

\subsubsection{Calculation of Releasing Load during Tunnel} Construction. When simulating the construction of tunnel, the equivalent node load of tunnel excavation boundary needs to be solved. The specific process is as follows: firstly, according to the initial stress acting on the tunnel excavation 


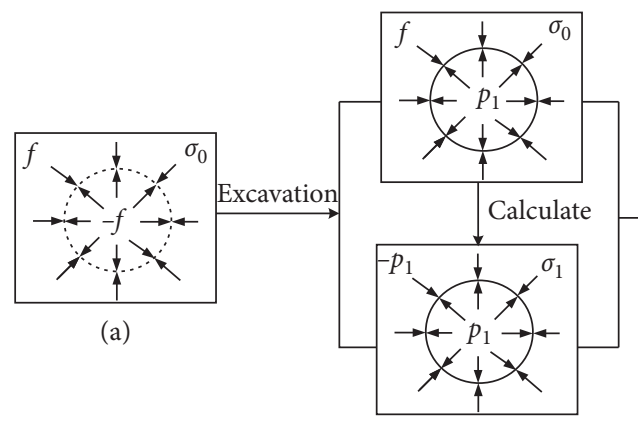

(b) Initial

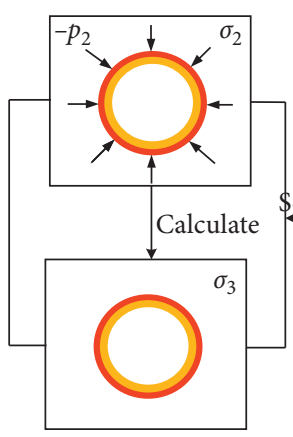

(d)

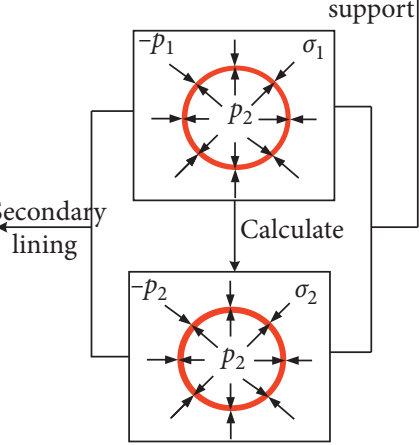

(c)

Figure 1: Schematic diagram of tunnel construction simulation by applying the virtual support force gradually release method.

boundary, the stress corresponding to the boundary node is solved by using the interpolation method; then the stress change between two adjacent boundaries is assumed to be linear; finally, according to the static equivalent principle, the total equivalent node load of each node on the tunnel excavation boundary is calculated. The schematic diagram is shown in Figure 2. Taking the boundary of tunnel excavation randomly, the node number is assumed to be $i$, and the equivalent release load caused by tunnel construction and excavation is defined as follows:

$$
\begin{aligned}
P_{x, i} & =\frac{1}{6}\left[2 \sigma_{x, i}\left(b_{1}+b_{2}\right)+\sigma_{x, i+1} b_{2}+\sigma_{x, i-1} b_{1}+2 \tau_{x y, i}\left(a_{1}+a_{2}\right)+\tau_{x y, i+1} a_{2}+\tau_{x y, i-1} a_{1}\right] \\
P_{y, i} & =\frac{1}{6}\left[2 \sigma_{y, i}\left(a_{1}+a_{2}\right)+\sigma_{y, i+1} a_{2}+\sigma_{y, i-1} a_{1}+2 \tau_{x y, i}\left(b_{1}+b_{2}\right)+\tau_{x y, i+1} b_{2}+\tau_{x y, i-1} b_{1}\right] \\
a_{1} & =x_{i-1}-x_{i} \\
a_{2} & =x_{i}-x_{i+1} \\
b_{1} & =y_{i}-y_{i-1} \\
b_{2} & =y_{i+1}-y_{i}
\end{aligned}
$$

where $\sigma_{x, i}, \sigma_{y, i}$, and $\tau_{x y, i}$ are the stress components at joint position $i$ before the tunnel excavation, respectively. $x$ and $y$ are the horizontal and vertical coordinates of the nodes, respectively.

\subsubsection{Selection of Constitutive Relationship of Surrounding} Rock of Tunnel. Surrounding rock material is a kind of complex mechanical material with plastic deformation. The yield strength of rock mass in compression is far greater than that in tension. With the increase of external load, stress state of surrounding rock material will change from elastic state to elastic-plastic state, which is called yield. The stress space formed by the transition critical stress state is called the yield surface. For general surrounding rock materials, the Drucker-Prager yield criterion can be used to determine the constitutive model of surrounding rock.

The Drucker-Prager yield criterion is a generalized von Mises yield criterion, which considers the effect of average hydrostatic pressure on yield. Since the criterion assumes 


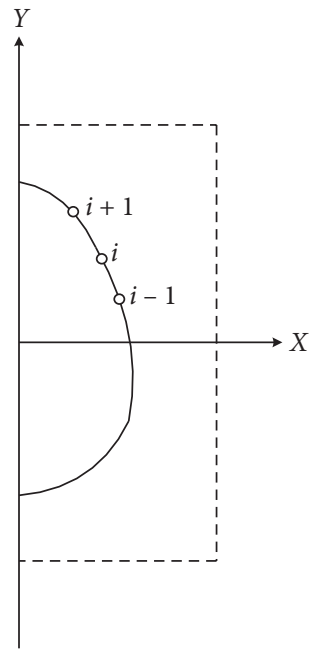

(a)

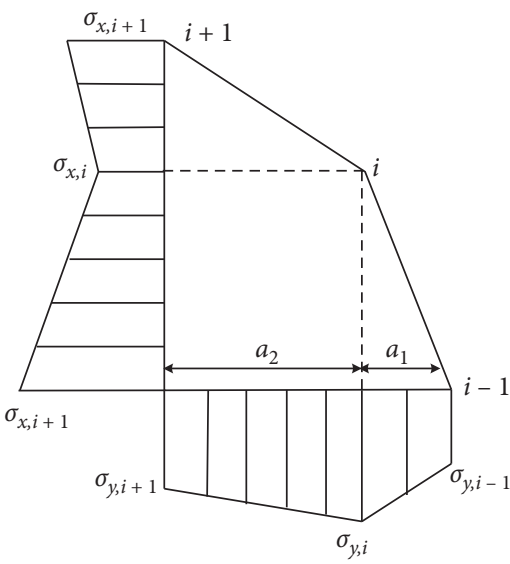

(b)

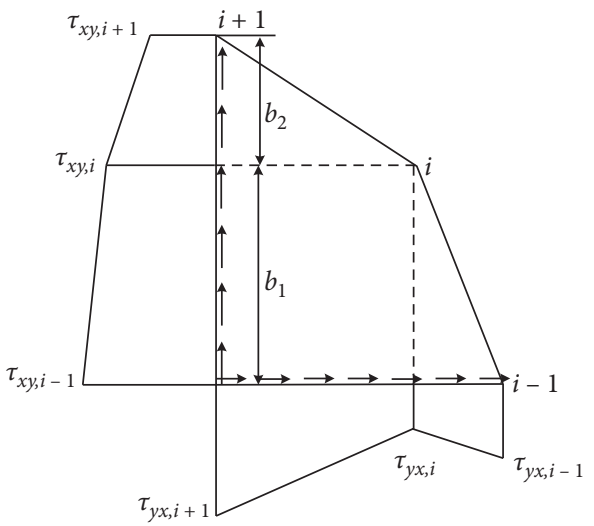

(c)

Figure 2: Schematic diagram for calculating the node forces at the tunnel excavation boundary. (a) Excavation boundary. (b) Equivalent load of normal stress. (c) Equivalent load of shear stress.

that the material is an ideal elastic-plastic material, its yield surface will not change with the state of material yielding or not, and its yield strength will increase with the increase of confining pressure (hydrostatic pressure). Its expression is described as follows:

$$
\begin{aligned}
F & =\sqrt{J_{2}}+\alpha I_{1}-k=0, \\
I_{1} & =\sigma_{x}+\sigma_{y}+\sigma_{z} \\
J_{2} & =\frac{1}{6}\left[\left(\sigma_{x}-\sigma_{y}\right)^{2}+\left(\sigma_{y}-\sigma_{z}\right)^{2}+\left(\sigma_{z}-\sigma_{x}\right)^{2}\right]+\tau_{y z}^{2}+\tau_{z x}^{2}+\tau_{x y}^{2}, \\
\alpha & =\frac{2 \sin \phi}{\sqrt{3}(3-\sin \phi)} \\
k & =\frac{6 c \cos \phi}{\sqrt{3}(3-\sin \phi)}
\end{aligned}
$$

where $F$ is the yield function and $I_{1}, J_{2}$ are the first invariant of stress and the second invariant of the stress bias tensor, respectively. $\phi, c$ are the angle of internal friction and cohesion of material, respectively. The yield surface of the Drucker-Prager yield criterion in the stress space is a conical surface, and its yield surface is shown in Figure 3.

\subsection{Structural Safety Evaluation of Tunnel Construction Using} the Reliability-Based Method. After establishing the FEM of tunnel, the control indexes of stress, strain, and displacement during the process of tunnel construction are obtained. The dynamic monitoring data of corresponding control indexes are measured following the construction process. Based on the dynamic monitoring data and the calculation results of FEM, the safety assessment of tunnel construction is implemented. Because some measured errors cannot be avoided completely, the structural safety evaluation method of tunnel construction is proposed based on the reliability theory.

Using the FEM of tunnel, a certain control index in the process of tunnel construction is obtained as follows:

$$
\mathbf{F}=\left\{F_{1}, F_{2}, F_{3}, \ldots, \quad F_{i}, \ldots, F_{N}\right\} .
$$

The monitoring data of corresponding indexes in the process of tunnel construction are obtained as follows:

$$
\mathbf{F}^{\prime}=\left\{F_{1}^{\prime}, F_{2}^{\prime}, F_{3}^{\prime}, \ldots, \quad F_{i}^{\prime}, \ldots, F_{N}^{\prime}\right\}
$$

Then, the ratio of the dynamic monitoring feedback obtained during the construction process to the calculated value of the finite element model is calculated by the following equation:

$$
\mathbf{S}=\left\{\frac{F_{1}^{\prime}}{F_{1}}, \frac{F_{2}^{\prime}}{F_{2}}, \frac{F_{3}^{\prime}}{F_{3}}, \ldots, \frac{F_{i}^{\prime}}{F_{i}}, \ldots \frac{F_{n}^{\prime}}{F_{n}}\right\}=\left\{S_{1}, S_{2}, S_{3}, \ldots, \quad S_{i}, \ldots S_{N}\right\} .
$$

It can be considered that vector $\mathbf{S}$ is the sample set of random variable $S$. The mean value and variance of random variable $S$ can be calculated as follows:

$$
\begin{aligned}
& E(S) \approx \bar{S}=\frac{1}{N} \sum_{i=1}^{N} S_{i}, \\
& \sigma^{2}(S) \approx \frac{1}{N-1} \sum_{i=1}^{N}\left(S_{i}-\bar{S}\right)^{2} .
\end{aligned}
$$

Let the reference ratio be a normal distribution random variable $R$ whose mean value and standard deviation are expressed as follows:

$$
\begin{aligned}
& E(R)=1, \\
& \sigma(R)=0.05 .
\end{aligned}
$$




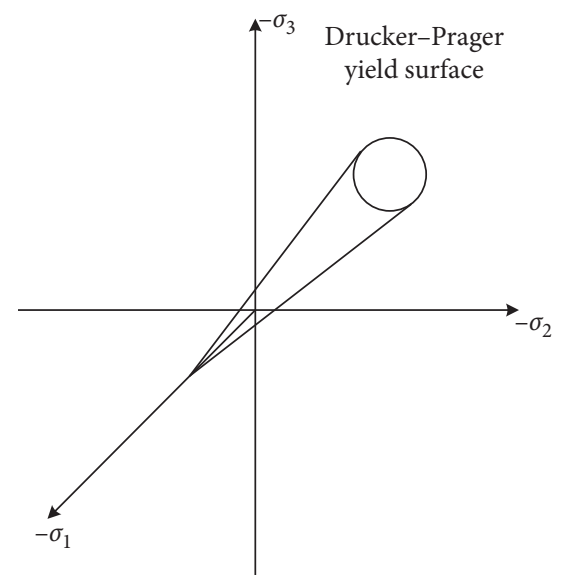

FiguRE 3: Yield surface of Drucker-Prager yield criterion.

To evaluate whether the structure fails or not, the limit state function is constructed as follows:

$$
Z=R-S \text {. }
$$

When $Z>0$, the structure is considered safe, and when $Z \leq 0$, the structure is considered to have safety risk. For ease of calculation, the following equations are defined:

$$
\begin{gathered}
{\left[X_{1}, X_{2}\right]=[R, S],} \\
Z=g(X)=X_{1}-X_{2},
\end{gathered}
$$

where $x_{i}$ is the value of the random variable array $\left[X_{1}, X_{2}\right]$. The advanced first-order second moment method is used to calculate the structural failure probability, and the specific steps are as follows [23]:

(i) Determining the initial value of the design point $x_{i}$ (the mean value is usually used as the initial iteration point, it means $x_{1}=M_{X}$ ) and assuming that the equation $i=1$.

(ii) Transforming $x_{i}$ into the standard normal random vector $y_{i}$, i.e., using the following relationship:

$$
y=T(x)=L^{-1} D^{-1}\left(x-M_{X}\right) .
$$

(iii) Changing the limit state function from $X$ space into $Y$ space, i.e.,

$$
G\left(y_{i}\right)=g\left(T^{-1}\left(y_{i}\right)\right)
$$

and then obtaining the probability transformation:

$$
x=T^{-1}(y)=D L y+M_{X} .
$$

(iv) Calculating the gradient vector $\nabla_{y_{i}} G$ of $G$ at the point $y_{i}$ by using the following equation:

$$
\nabla_{y} G=\left[J_{X Y}(x)\right]^{T} \cdot \nabla_{x} g .
$$

(v) Calculating the normal vector $\alpha_{i}$ at the point $y_{i}$ by using the following equation:

$$
\alpha_{i}=-\frac{\nabla_{y_{i}} G}{\left\|\nabla_{y_{i}} G\right\|} \text {. }
$$

(vi) Calculating the reliability index at the point $y_{i}$, i.e., $\beta_{i}=\alpha_{i}^{T} y_{i}$

(vii) $i=i+1$, and then obtaining $y_{i+1}$ by using the following equation:

$$
y_{i+1}=\left[\frac{G\left(y_{i}\right)}{\left\|\nabla_{y_{i}} G\right\|}+\alpha_{i}^{T} y_{i}\right] \alpha_{i} .
$$

(viii) Repeating step (ii) to step (vii) until $\beta_{i}$ or $y_{i}$ converges.

(ix) Calculating an approximation of the probability of failure:

$$
p_{f} \approx \Phi\left(-\beta_{i}\right)
$$

\section{Numerical Analysis of the Construction Process of an Actual Shallow-Buried Tunnel with Underground Excavation}

3.1. Brief Description of Yuhan Road Tunnel. Yuhan road tunnel belongs to the second stage of southern extension line of Shunhe Viaduct project, which is an important part of Jinan rapid road network planning system, in China. The tunnel is arranged along Yuhan road, with a length of about $2.3 \mathrm{~km}$. Considering the influence of geological conditions, the pipeline buried above, the buildings along the tunnel, and other factors, the buried depth of the tunnel is between $6 \mathrm{~m}$ and $10 \mathrm{~m}$ along the longitudinal direction. The maximum longitudinal slope of the tunnel is $2.6 \%$ and the minimum longitudinal slope is $0.9 \%$. The scene photos of Yuhan road tunnel are shown in Figure 4.

The mileage pile numbers of the tunnel are between $\mathrm{ZK} 1+325$ and $\mathrm{ZK} 1+405$, and the basic tunnel form of this section is neighboring tunnel. According to relevant geological survey and design data, the properties of geomaterial used in the FEM are listed in Table 1. 


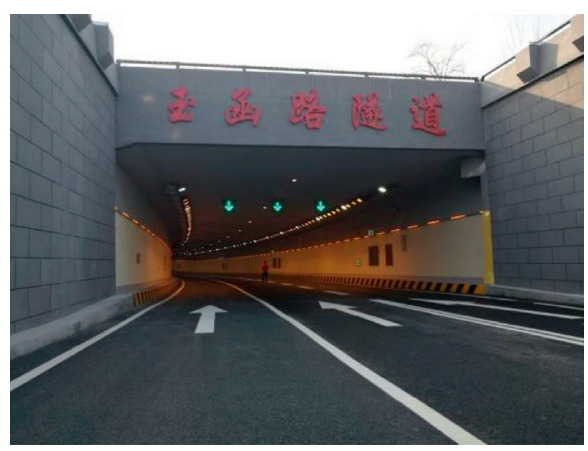

(a)

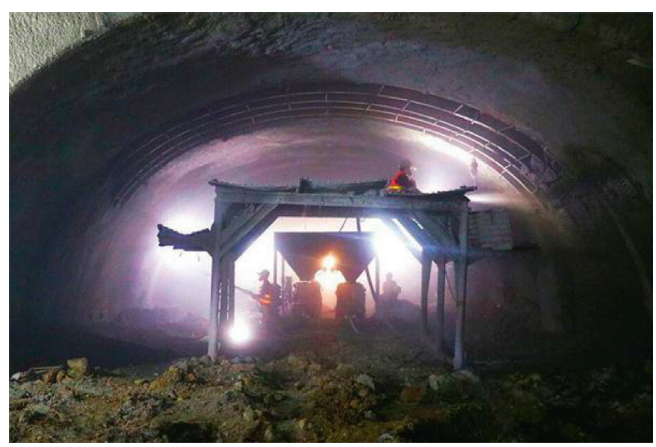

(b)

Figure 4: Scene photos of Yuhan road tunnel.

TAble 1: Properties of geomaterial used in the tunnel model.

\begin{tabular}{lccccc}
\hline Material & Density $\left(\mathrm{kg} / \mathrm{m}^{3}\right)$ & Poisson's ratio & Elastic modulus $(\mathrm{MPa})$ & Cohesion $(\mathrm{kPa})$ & Internal friction angle $\left(^{\circ}\right)$ \\
\hline Clay & 1900 & 0.36 & 36.8 & 15 & 28 \\
Limestone & 2000 & 0.35 & 700 & 310 & 25 \\
Marlite rock & 2100 & 0.35 & 1100 & 208 & 27 \\
Anchorage zone & 2040 & 0.32 & 310 & $/$ & 30 \\
Initial support & 2400 & 0.2 & 36200 & $/$ & $/$ \\
Inverted arch & 2400 & 0.2 & & $/$ \\
\hline
\end{tabular}

During the process of tunnel excavation, the tunnel section is divided into four parts according to the actual construction plan, which is shown in Figure 5. The step construction method is adopted, and the excavating sequence of each part is carried out in numerical order. Considering the interaction effect of adjacent tunnel face, the longitudinal excavation process of neighboring tunnel should be separated, as shown in Figure 6. To investigate the internal force and deformation during construction, the actual specific construction process is simulated step by step in FE software. During the simulation, in order to reduce the huge computation, the corresponding construction steps are simplified.

3.2. Generation of the FEM of Yuhan Road Tunnel. Ansys is adopted to simulate the construction process, and the following assumptions are made in the construction simulation:

(i) Assuming the surface ground is flat, since the site terrain undulation is small.

(ii) Only the ground stress caused by dead weight is considered in the calculation of initial ground stress, since the tunnel is ultra-shallow-buried tunnel, and the tectonic secondary stress caused by deficient geological condition is ignored.

(iii) The Drucker-Prager model is used for the constitutive model of surrounding rock of the tunnel, and the initial support is assumed as elastic.

(iv) Assuming that only part of the stress is released at the start of excavation and the residual ground stress is released after the initial support is applied, since the tunnel is constructed by shallow-buried tunneling method.

Previous research studies show that, during the process of tunnel excavation, only the surrounding rock within a certain range is affected, and the stress and displacement of the surrounding rock are hardly changed. Under normal circumstances, the influence of tunnel excavation on surrounding rock is less than $5 \%$ within 3 times the diameter of the tunnel and less than $1 \%$ within 5 times the diameter of the tunnel. In order to minimize the interference of boundary conditions on the analysis results and reduce the calculation amount, the calculation range is 4 times the hole diameter in this model, the model width is $80 \mathrm{~m}$, the bottom boundary of the model is $20 \mathrm{~m}$ below the tunnel, the upper side of the model is a flat surface, and the longitudinal length of the model is $16 \mathrm{~m}$.

When the FEM of tunnel is established, the surrounding rock, bolt, and middle rock wall are simulated by solid 45 element, the tunnel support structures (temporary support, invert, and initial support) are simulated by shell 63 element, and mesh200 element is used to divide mesh grid, which helps generate the three-dimensional model.

The boundary conditions applied to the tunnel are as follows: $X$ direction constraint is applied to the left and right boundaries; $Y$ direction constraint is applied to the lower boundary; the upper surface is set as a free boundary; and $Z$ direction constraint is applied to front and back boundaries along the tunnel. The reasons for the above constraints on the tunnel boundary are described as follows: the left and right boundaries are far apart, and thus the excavation will not cause displacement of the boundary in the $X$ direction, 

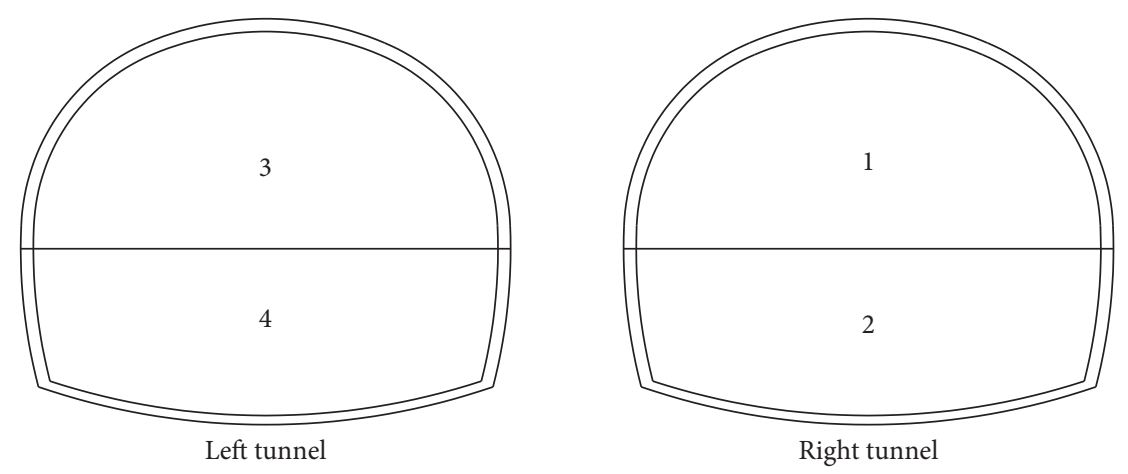

Figure 5: Process diagram of tunnel section excavation.

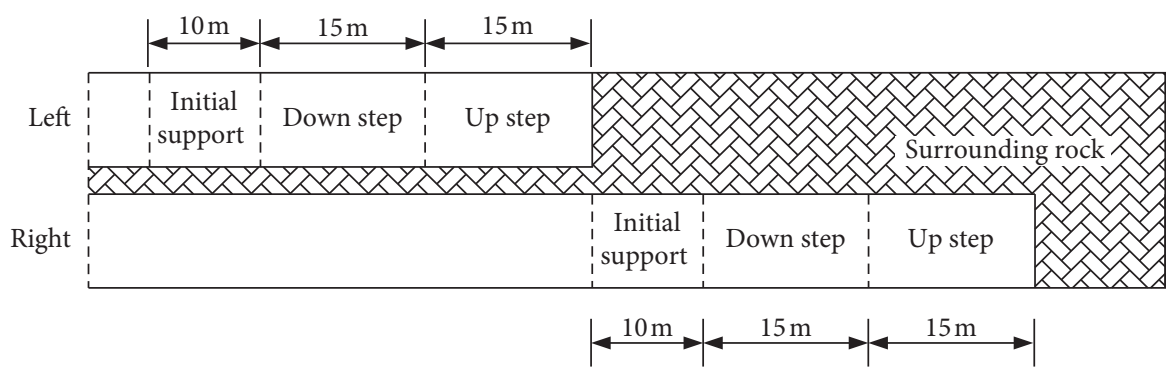

FIgURE 6: Process diagram of tunnel longitudinal excavation.

and the settlement only occurs in the $Y$ direction due to dead load; since the excavation process does not affect the $Y$ direction displacement of the lower boundary, the $Y$ direction of the lower boundary is constrained; the upper boundary is ground surface, and its horizontal and vertical displacement is greatly affected by tunnel excavation, and thus it is set as a free boundary. The FEM is established with 157811 nodes and 130366 elements, as shown in Figure 7.

\subsection{Results of Finite Element Analysis of Yuhan Road Tunnel}

3.3.1. Deformation Analysis of the Neighboring Tunnel during the Construction. The deformation nephogram of surrounding rock during the construction process is shown in Figure 8.

As shown in Figure 8, during the construction process, the vertical displacement distribution of the tunnel is relatively symmetrical with grade V1 surrounding rock, and settlement occurs at the arch roof, the step, and arch bottom of the tunnel bulges. At this stage of construction process, the maximum $Y$ direction displacement reaches $4.9 \mathrm{~mm}$, and the maximum displacement in the cross section reaches $1.7 \mathrm{~mm}$

3.3.2. Stress Analysis of the Neighboring Tunnel during the Construction. The stress nephogram of surrounding rock of tunnel during the construction process is shown in Figure 9.

As shown in Figure 9, the stress state of surrounding rock is in the form of symmetry. This is because during the process of neighboring tunnel construction, the left and right pilot tunnels are rarely influenced by each other, and the disturbance to surrounding rock is small; there is little stress concentration. At this stage of construction process, the maximum tensile stress in surrounding rock is $0.2 \mathrm{MPa}$, while the maximum compressive stress is $1.48 \mathrm{MPa}$.

3.3.3. Deformation Analysis of the Neighboring Tunnel after the Construction. The deformation nephogram of surrounding rock after the construction process is shown in Figure 10 .

It can be seen from Figure 10 that, after completing the tunnel excavation, the maximum $Y$ direction displacement is $4.9 \mathrm{~mm}$, which occurs at the arch top of the left and right pilot tunnels; there is uplift at the arch bottom of the tunnel, and the maximum uplift displacement is $2.7 \mathrm{~mm}$. Since the left and right pilot tunnels have little influence on each other during the tunnel construction, the displacement nephogram of the left and right tunnels is close to symmetry. The largest positive displacement in $X$ direction of the tunnel is located at the top right of the right pilot tunnel, with a maximum value of $1.8 \mathrm{~mm}$; the largest positive displacement in $X$ direction of the tunnel is located at the top left of the left pilot tunnel, with a maximum value of $1.9 \mathrm{~mm}$.

3.3.4. Stress Analysis of the Neighboring Tunnel after the Construction. The stress nephogram of surrounding rock after the construction process is shown in Figure 11.

It can be shown from Figure 11 that the maximum first principal stress of the surrounding rock is $0.28 \mathrm{MPa}$, and the maximum tensile stress occurs at the vault of the left tunnel, 


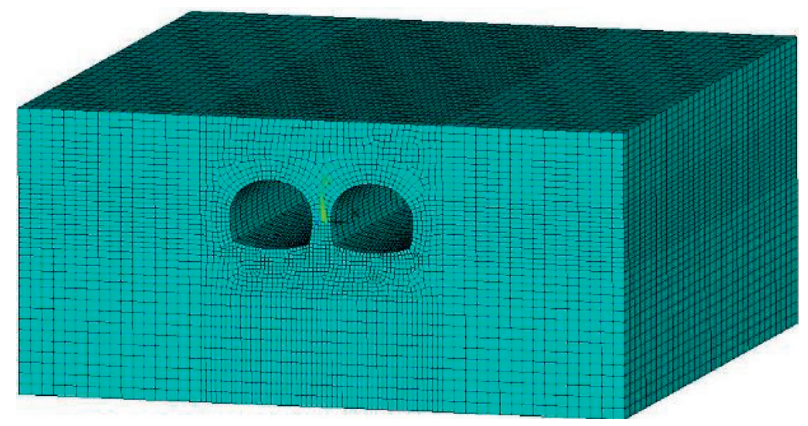

(a)

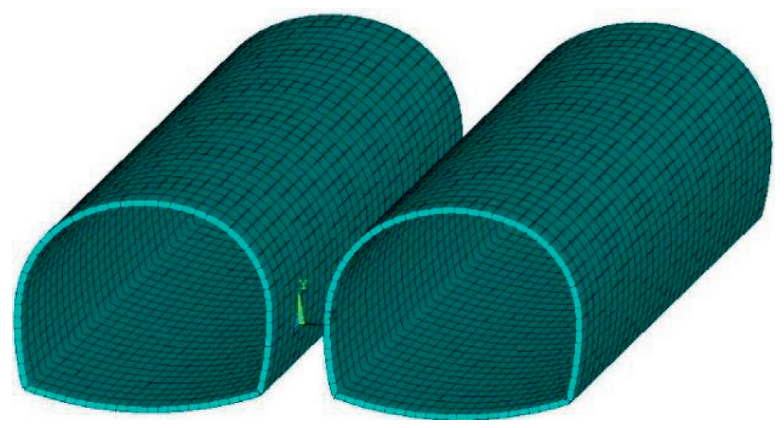

(b)

Figure 7: Model element plots of the neighboring tunnel. (a) Element of the surrounding rock. (b) Element of the initial support.

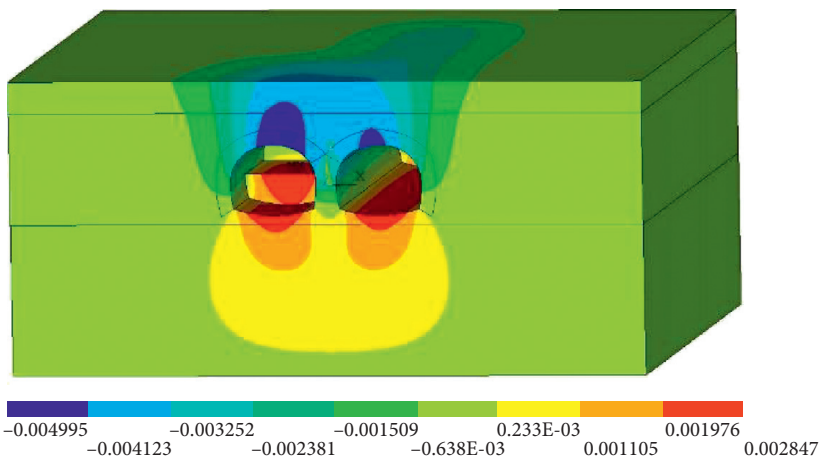

(a)

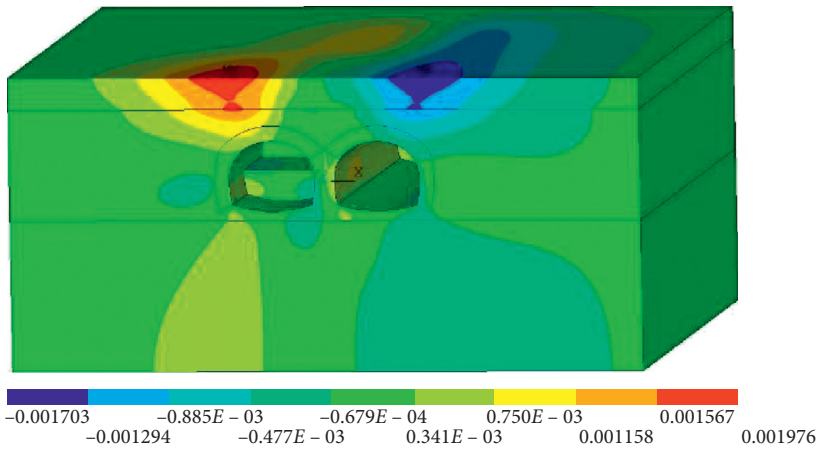

(b)

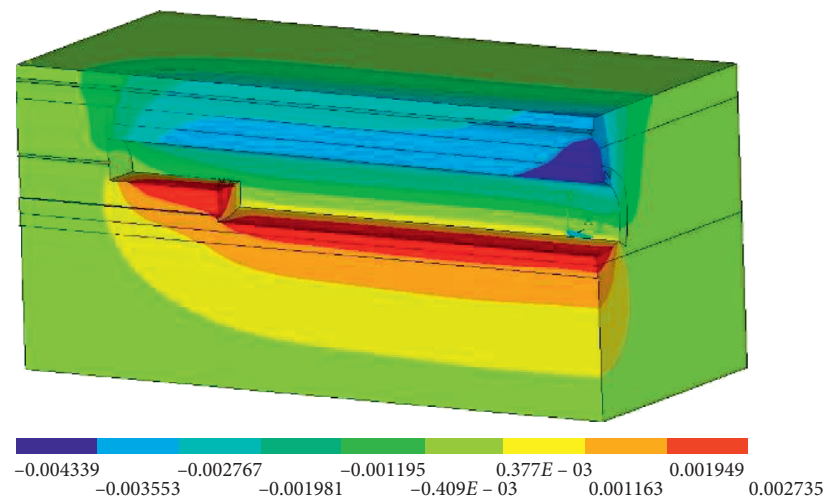

(c)

FIGURE 8: Deformation nephogram of surrounding rock of tunnel during the construction process. (a) Deformation nephogram along Yaxis. (b) Deformation nephogram along $X$-axis. (c) Deformation nephogram of right tunnel section along $Y$-axis.

and thus there is a need to pay attention to the surrounding rock when excavating this area. The minimum third principal stress of the surrounding rock is $-1.49 \mathrm{MPa}$, which appears at the arch foot of the left tunnel, where stress concentration phenomenon occurs in this spot and sufficient attention must be paid in the construction process.

\section{Safety Evaluation of an Actual Shallow-Buried Tunnel with Underground Excavation during Construction}

Through the above numerical simulation results, it can be seen that the deformation and stress of Yuhan road tunnel during the construction process are small, which meets the 


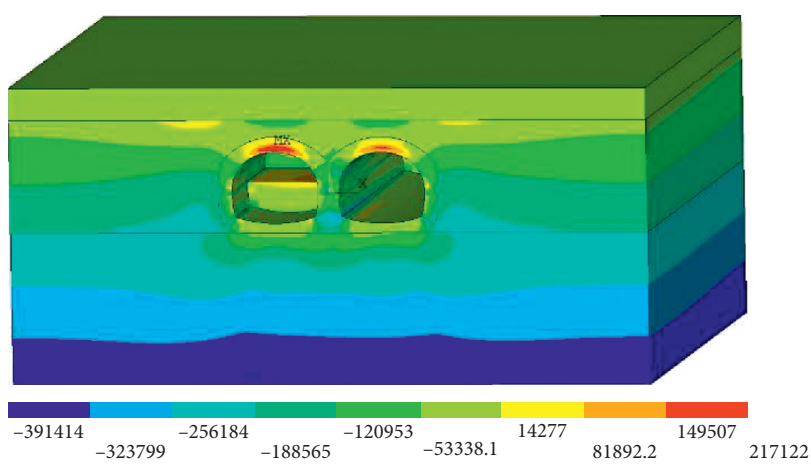

(a)

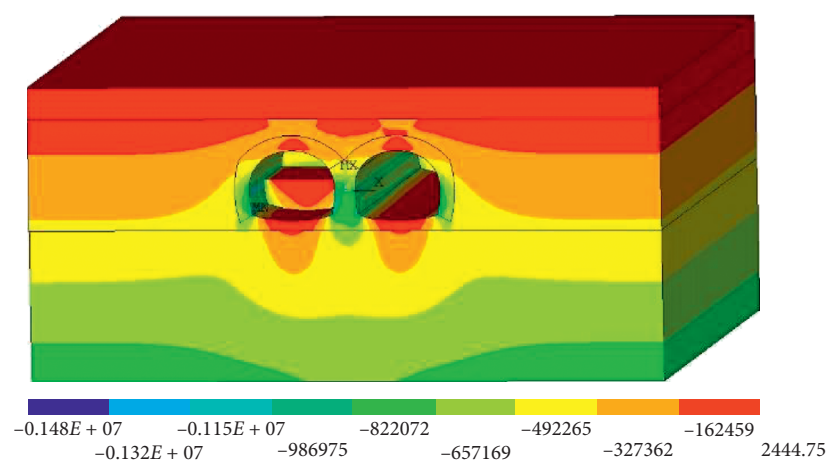

(b)

FIGURE 9: Stress nephogram of surrounding rock during the construction process. (a) First principal stress nephogram. (b) Third principal stress nephogram.

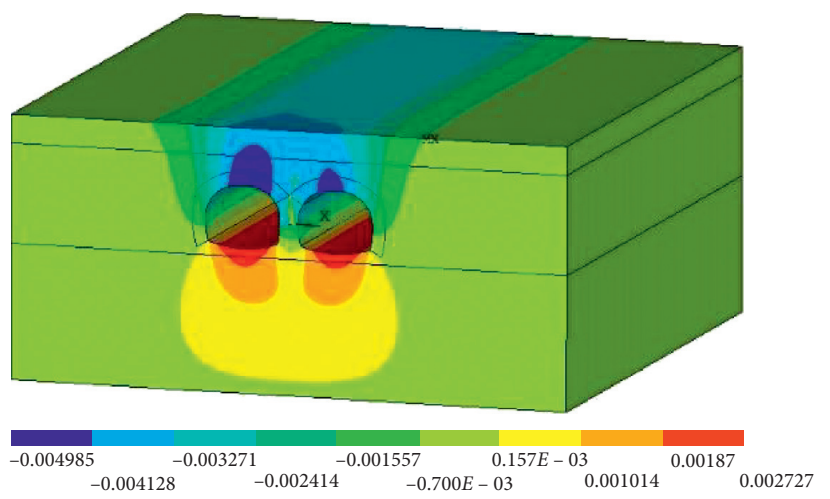

(a)
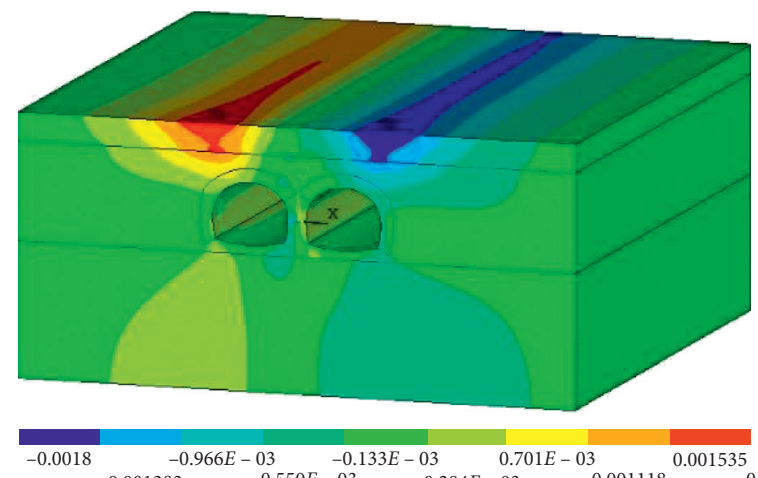

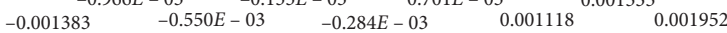

(b)

Figure 10: Deformation nephogram of surrounding rock after the construction process. (a) Deformation nephogram along $Y$-axis. (b) Deformation nephogram along $X$-axis.

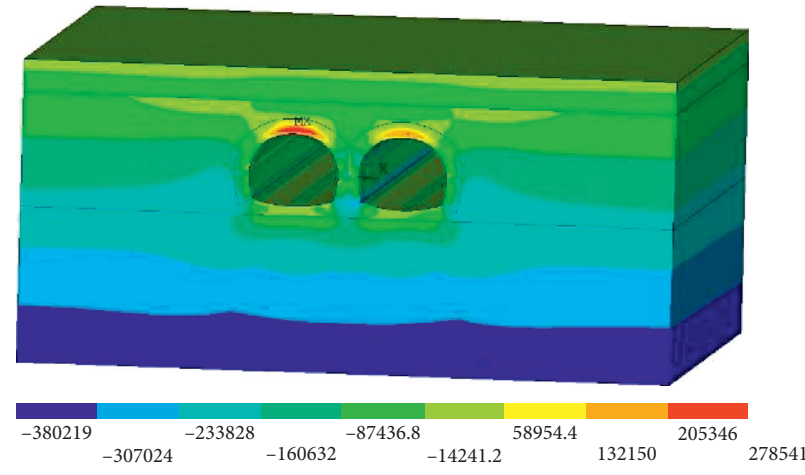

(a)

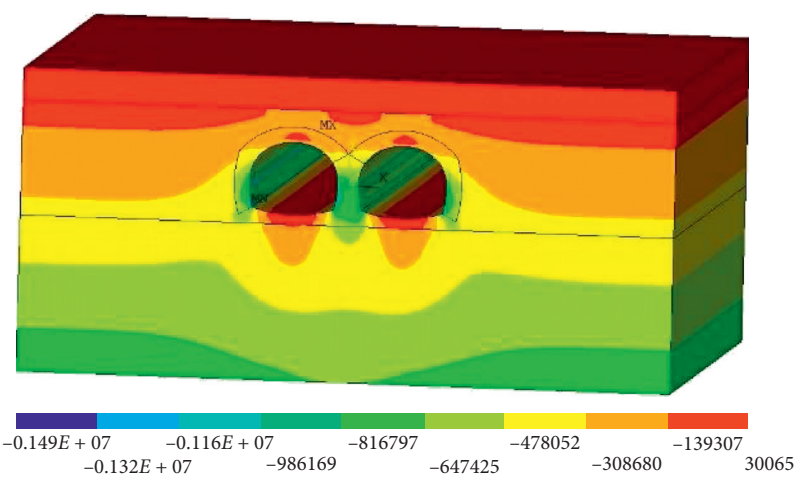

(b)

FIGURE 11: Stress nephogram of surrounding rock after the construction process. (a) First principal stress nephogram. (b) Third principal stress nephogram.

requirements of relevant specifications. The stress does not exceed the bearing capacity of surrounding rock of tunnel and concrete materials, so it can be judged that the tunnel construction scheme is feasible. In order to further ensure the safety of tunnel construction, the vertical displacement at the top of the tunnel was monitored during the actual tunnel construction.
The comparison between the measured data and the calculated results of FEM analysis is shown in Figures 12 and 13.

Based on the measured dynamic monitoring data and the established FEM, the construction safety of this tunnel is evaluated. The failure probability of different construction stages after the $10^{\text {th }}$ load step is shown in Figure 14. It is 


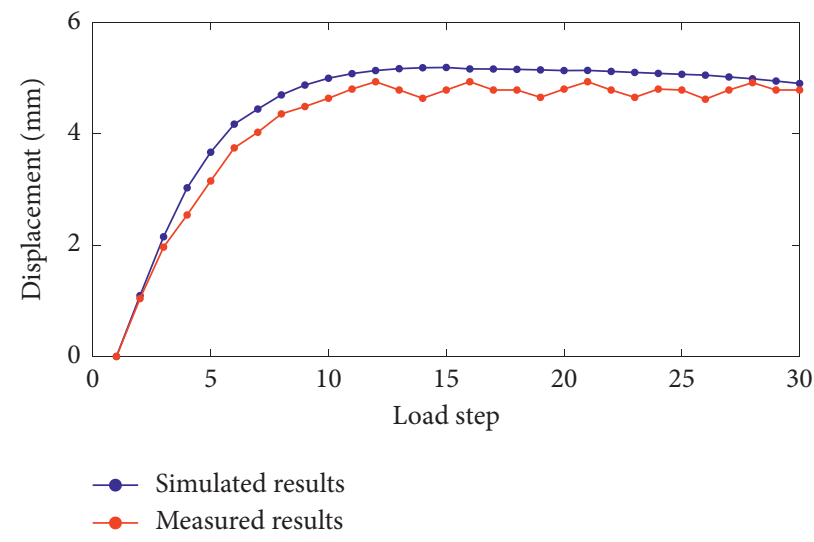

FIGURE 12: Vertical displacement diagram of vault.

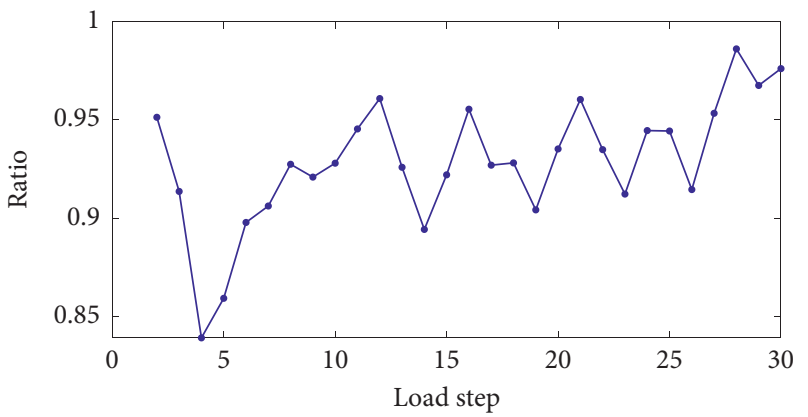

FIGURE 13: The ratio of monitoring data to numerical simulation results.

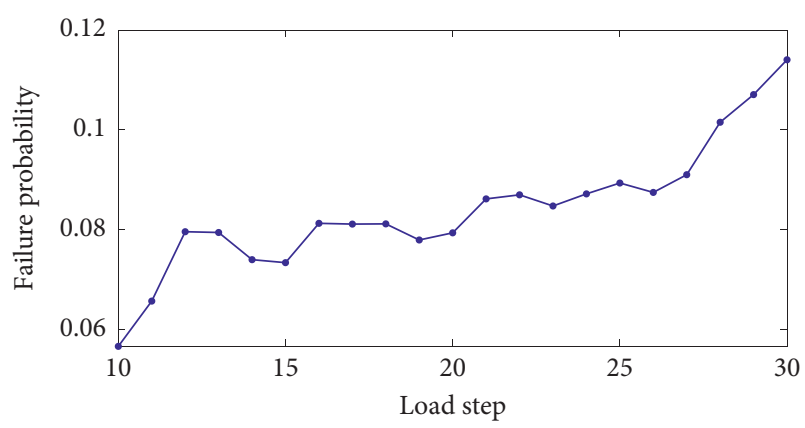

FIGURE 14: Failure probability of structure during construction.

deduced that the failure probability of the structure is very small, which is consistent with the actual situation of construction; thus, the proposed method is effective.

\section{Conclusions}

In this study, a reliability-based method is proposed to evaluate the structural safety of tunnel by using the dynamic monitoring data during construction. The following conclusions are drawn:

(i) The measured results show that the method of modelling the tunnel excavation of Yuhan road tunnel is effective, and the accuracy of the analytical results satisfies the engineering requirements. (ii) The proposed reliability-based method is effective to evaluate the structural safety of actual tunnel during the process of construction.

(iii) It is necessary to combine the analytical simulation and prediction with the dynamic monitoring data in order to calculate the failure probability of tunnel during the whole process of construction.

\section{Data Availability}

The data used to support the findings of this study are included within the article.

\section{Conflicts of Interest}

The authors declare that there are no conflicts of interest regarding the publication of this paper.

\section{Acknowledgments}

This study was supported by the Key Research \& Development Program of Shandong Province of China (grant no: 2019JZZY010427).

\section{References}

[1] A. Prosek and M. Cepin, "Success criteria time windows of operator actions using RELAP5/MOD3.3 within human 
reliability analysis," Journal of Loss Prevention in The Process Industries, vol. 21, no. 3, pp. 260-267, 2008.

[2] S. Chi and C. H. Caldas, "Image-based safety assessment: automated spatial safety risk identification of earthmoving and surface mining activities," Journal of Construction Engineering and Management, vol. 138, no. 3, pp. 341-351, 2012.

[3] Z. Lan and M. Huang, "Safety assessment for seawall based on constrained maximum entropy projection pursuit model," Natural Hazards, vol. 91, no. 3, pp. 1165-1178, 2018.

[4] M. Yang, "System safety assessment using safety entropy," Journal of Loss Prevention in The Process Industries, vol. 66, Article ID 104174, 2020.

[5] C. Xin and B. Gao, "Construction Methods for Long-Span and Bifurcation Tunnel Structure," Applied Mechanics And Materials, vol. 580-583, pp. 983-986, 2014.

[6] Y. Zhang, J. Yang, and F. Yang, "Field investigation and numerical analysis of landslide induced by tunneling," Engineering Failure Analysis, vol. 47, pp. 25-33, 2015.

[7] B. Jin, Y. Liu, C. Yang, Z. Tan, and J. Zhang, "Construction technique of long-span shallow-buried tunnel considering the optimal sequence of pilot-tunnel excavation," Advances in Materials Science and Engineering, vol. 2015, Article ID 491689, 2015.

[8] R. Keutgen and J.-L. Lilien, "Benchmark cases for galloping with results obtained from wind tunnel facilities validation of a finite element model," IEEE Transactions on Power Delivery, vol. 15, no. 1, pp. 367-374, 2000.

[9] G. Degrande, D. Clouteau, R. Othman et al., "A numerical model for ground-borne vibrations from underground railway traffic based on a periodic finite element-boundary element formulation," Journal of Sound and Vibration, vol. 193, no. 3-5, pp. 645-666, 2006.

[10] P. G. C. Prazeres, K. Thoeni, and G. Beer, "Nonlinear analysis of NATM tunnel construction with the boundary element method," Computers and Geotechnics, vol. 40, pp. 160-173, 2012.

[11] H. Yoshimura, T. Yuki, Y. Yamada, and N. Kokubun, "7. Analysis and monitoring of the Miyana railway tunnel constructed using the New Austrian tunnelling method," International Journal of Rock Mechanics and Mining Sciences \& Geomechanics Abstracts, vol. 23, no. 1, pp. 67-75, 1986.

[12] J. Sulem, M. Panet, A. Guenot et al., "An analytical solution for time-dependent displacements in a circular tunnel," International Journal of Rock Mechanics and Mining Sciences \& Geomechanics Abstracts, vol. 24, no. 3, pp. 155-164, 1987.

[13] A. J. Abbo, D. W. Wilson, S. W. Sloan, and A. V. Lyamin, "Undrained stability of wide rectangular tunnels," Computers and Geotechnics, vol. 53, pp. 46-59, 2013.

[14] H.-T. Yu, Y. Yuan, X. Liu, Y.-W. Li, and S.-W. Ji, "Damages of the Shaohuoping road tunnel near the epicentre," Structure and Infrastructure Engineering, vol. 9, no. 9, pp. 935-951, 2013.

[15] H. M. Shahin, T. Nakai, K. Ishii, T. Iwata, and S. Kuroi, "Investigation of influence of tunneling on existing building and tunnel: model tests and numerical simulations," Acta Geotechnica, vol. 11, no. 3, pp. 679-692, 2016.

[16] A. Alsahly, J. Stascheit, and G. Meschke, "Advanced finite element modeling of excavation and advancement processes in mechanized tunneling," Advances in Engineering Software, vol. 100, pp. 198-214, 2016.

[17] A. Ali, A. V. Lyamin, J. Huang, S. W. Sloan, and M. J. Cassidy, "Undrained stability of a single circular tunnel in spatially variable soil subjected to surcharge loading," Computers and Geotechnics, vol. 84, pp. 16-27, 2017.
[18] G. Zheng, J. Sun, T. Zhang, Q. Fan, Y. Diao, and H. Zhou, "Eulerian finite element model for stability analysis of circular tunnels in undrained clay," Engineering Failure Analysis, vol. 91, pp. 216-224, 2018.

[19] J. Shiau and F. Al-Asadi, "Determination of critical tunnel heading pressures using stability factors," Computers and Geotechnics, vol. 119, Article ID 103345, 2020.

[20] J. P. Ou and H. Li, "Structural health monitoring in mainland China: review and future trends," Structural Health Monitoring, vol. 9, pp. 219-231, 2010.

[21] V. G. M. Annamdas, S. Bhalla, and C. K. Soh, "Applications of structural health monitoring technology in Asia," Structural Health Monitoring: An International Journal, vol. 16, no. 3, pp. 324-346, 2017.

[22] S. Zhang and Y. Liu, "Damage detection of bridges monitored within one cluster based on the residual between the $\mathrm{cu}$ mulative distribution functions of strain monitoring data," Structural Health Monitoring, vol. 19, no. 6, pp. 1764-1789, 2020.

[23] R. E. Melchers and A. T. Beck, Structural Reliability Analysis and Prediction, pp. 95-112, John Wiley and Sons, London, UK, 3rd edition, 2018. 